\section{CME: Case reports}

February's CME consists of a series of case reports that have been received over the past 12 months. Many journals, local and international, feature case reports within their pages, and younger doctors in particular are encouraged to write up their more interesting cases in this format.

According to Wikipedia, in medicine, a case report is a detailed report of the symptoms, signs, diagnosis, treatment and follow-up of an individual patient'. Case reports are usually written to provide an unusual or novel occurrence of a set of signs and symptoms, or, as is the case in some of the reports published this month, unusual presentations of a particular disease entity. Case reports often contain some kind of literature review of other reported cases, even if only to say that the report is of a rare occurrence.

Case reports are, by their very nature, anecdotal and are placed at the foot of the hierarchy of clinical evidence, together with case series. However, case reports are usually thought to have genuinely useful roles in medical research and in evidence-based medicine.

However, one of the most useful roles of case reports is that of medical education, both formally, providing a structure for casebased learning (which we all did at medical school), and informally, for the general reader. In both cases, interesting and unusual presentations are helpful to day-to-day practice and will often trigger recognition of a diagnosis or pathology in a puzzling clinical case.

All the case reports presented in this issue of CME are local, and selected for their particular usefulness to our younger and less experienced colleagues.

\section{Renal disease and haemodialysis in HIV-positive patients}

An article on morbidity and mortality of black HIV-positive patients with end-stage kidney disease receiving chronic haemodialysis in South Africa (SA) $)^{[1]}$ and an accompanying editorial ${ }^{[2]}$ reveal the extent of renal disease in the HIV-positive population. Renal disease affects up to $30 \%$ of HIV-infected patients. HIV-associated nephropathy (HIVAN) is most common and, unless treated with antiretroviral therapy (ART), progresses rapidly to end-stage renal disease (ESRD). ESRD is projected to increase further now that HIV-positive patients are living longer on ART and are increasingly manifesting the diseases of lifestyle, including hypertension and diabetes.

All HIV-positive patients should be screened for chronic kidney disease at first encounter with any health service. This is particularly important in view of the fact that HIVAN can occur with high CD4 counts. Screening should include urinalysis and measurement of kidney function. Patients manifesting renal involvement should be fast-tracked for ART. Furthermore, being HIV-positive is no longer a contraindication to renal transplantation, provided patients are established on ART and achieve acceptable CD4 counts and suppressed viral loads.

\section{Linking cervical cancer screening to human papillomavirus (HPV) vaccination}

This issue of $S A M J$ features the second article on the Vaccine and Cervical Cancer Screen (VACCS) project, on linking cervical cancer screening to HPV vaccination in the South-West District of Tshwane, Gauteng, SA. ${ }^{[3]}$ This study provided the novel opportunity to investigate the outcome of cervical cancer screening in mothers and guardians by linking this to the vaccination of the grade $4-7$ girls in their care. New molecular screening technology was utilised, permitting self-sampling in a home setting with a screen kit offered to female parents and guardians (plus an extra one for a friend or family member). The screen kit consisted of a tampon with user instructions: women inserted the tampon vaginally and removed it after one hour. The used tampon was placed in a container with buffer and, together with personal information, was returned to the school in a sealed envelope. DNA 


\section{EDITOR'S CHOICE}

was extracted from the tampon specimens and tested for any of the 15 high-risk viral types $(16,18,31,33,35,39,45,51,52,56,58,59,68,73$, 82), HPV 16 and 18 being the two most oncogenic. Roughly half of the women took up the screening opportunity, and molecular screening identified cervical cancer risk in $30 \%$ and a high risk of future disease in $9.1 \%$. Using the school infrastructure as well as mobile phone technology, all women received their screen results.

This is an important development, given the limited success registered by the national cervical cancer prevention programme, launched in 2000, which offers three Papanicolaou smears per lifetime (starting after the age of 30 , at 10 -year intervals).

\section{Digitalis reappraised}

Still here today, but gone tomorrow? Opi ${ }^{[4]}$ suggests that there are very few arguments left in favour of the use of digitalis in the control of heart rate in atrial fibrillation. Following negative mortality data from one large recent study of digitalis in heart failure (HF), enthusiasm for further testing for the benefit of digitalis that would necessitate a large, multicentre, prospective randomised controlled trial is waning. Opie suggests that digoxin, for the indication of HF, would not be passed by regulatory agencies on the basis of present data. (See also in CME 'Digoxin therapy in the modern management of cardiovascular disease: An unusual but serious complication ${ }^{[5]}$.)

\section{Why is cancer not a priority in South Africa?}

An editorial asks the above question. ${ }^{[6]}$ The National Cancer Registry (NCR) is an invaluable source of cancer data for the country. Established in 1986 as a voluntary, pathology-based cancer reporting system, the Registry within the National Health Laboratory Service is the principal cancer surveillance system in SA. Regulation 380 of the 2011 National Health Act formally established the NCR as the main cancer surveillance agency and mandated reporting of all confirmed cancers in SA to the NCR.

The NCR receives over 100000 cancer reports annually; approximately 80000 are new cases, on the basis of which cancer incidence is calculated. Registry data have been used to highlight cancers of importance in the SA context. Data from the Johannesburg Cancer Case Control Study (JCCCS), conducted by the Cancer Epidemiology Research Group, have been used to extensively describe the epidemiology of HIV-related cancers and particularly to explore the relationship between Kaposi's sarcoma and HIV. The JCCCS has also contributed to risk factor analysis in the International Collaboration of Epidemiological Studies of Cervical Cancer.

The NCR manages cancer surveillance in the context of SA's dual health system, comprising a large public health infrastructure serving approximately $84 \%$ of the population and a smaller private health system catering to $16 \%$. It is dismaying to learn from Singh et al. ${ }^{[7]}$ that private laboratory cancer data reporting, which was consistent throughout the early 2000s, was withheld from 2005 to 2007, resulting in a $28 \%$ under-reporting from private healthcare centres (see Fig. 1, reproduced below). Fortunately the impact of withheld private data appears to have been minimal in that there was only a $4 \%$ decrease in overall cancer reporting, reflecting the reality that four out of every five SA citizens receive care in public healthcare systems. Fortunately, too, relationships with private sector laboratories have been renewed and a standard system has been established to receive private sector pathology data electronically.

In an era of growing prioritisation of NCDs and with global cancer burdens estimated to increase significantly, the NCR has an invaluable role to play in the health and health planning landscape of SA. In view of the progressive health developments in the country,

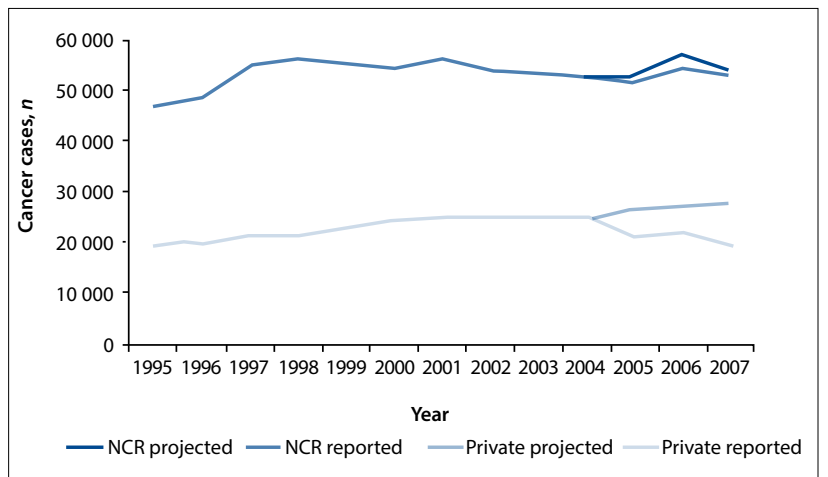

Fig. 1. Actual and projected case reporting from private laboratories and to the NCR, 1995 - 2007.

such as the introduction of National Health Insurance, there is an imperative to accurately quantify the cancer burden, and thus the cost of cancer services to be provided to the SA population.

\section{Non-communicable diseases (NCDs)}

Two articles in this issue address comorbidity and multimorbidity in NCDs in the SA setting, ${ }^{[8,9]}$ the former suggesting that future clinical guidelines, training of primary care nurses and involvement of doctors in the continuum of care should address the complexity of patients with NCDs and multimorbidity, and the latter warning against mobilisation of scarce resources to implement mass screening for diabetes and hypertension in the absence of adequate evidence of benefit.

As is well recognised, the SA healthcare system faces a quadruple burden of disease, characterised by HIV/AIDS and tuberculosis, injury and violence, maternal and child health issues and NCDs. The World Health Organization estimates the burden of NCDs to be two to three times higher in SA than in high-income countries. NCDs are estimated to contribute $28 \%$ to the total burden of disease, and this is predicted to increase substantially over the next few decades. In the Western Cape, NCDs account for five of the ten leading causes of death: ischaemic heart disease, diabetes, cerebrovascular disease, lung cancer and chronic obstructive pulmonary disease. NCD distribution reflects socioeconomic disparities, with the heaviest burden among poor communities in urban areas, posing a developmental challenge to the country. ${ }^{[10]}$

JS

1. Fabian J, Maher HA, Clark C, Naicker S, Becker P, Venter WDF. Morbidity and mortality of black HIVpositive patients with end-stage kidney disease receiving chronic haemodialysis in South Africa. S Afr Med J 2015;105(2):110-114. [http://dx.doi.org/10.7196/SAMJ.8369]

2. Wearne N. Morbidity and mortality of black HIV-positive patients with end-stage kidney disease receiving chronic haemodialysis in South Africa. S Afr Med J 2015;105(2):105-106. [http://dx.doi. org/10.7196/SAMJ.9068]

3. Snyman LC, Dreyer G, Botha MH, van der Merwe FH, Becker PJ. The Vaccine and Cervical Cancer Screen (VACCS) project: Linking cervical cancer screening to HPV vaccination in the South-West District of Tshwane, Gauteng, South Africa. S Afr Med J 2015;105(2):115-120. [http://dx.doi.org/10.7196/ SAMJ.8418]

4. Opie LH. Digitalis reappraised: Still here today, but gone tomorrow? S Afr Med J 2015;105(2):88-89. [http://dx.doi.org/10.7196/SAMJ.8634]

5. Mkoko P, Mokhele N, Ntsekhe M, Ntusi NBA. Digoxin therapy in the modern management of 5. Mkoko P, Mokhele N, Ntsekhe M, Ntusi NBA. Digoxin therapy in the modern management of
cardiovascular disease: An unusual but serious complication. S Afr Med J 2015;105(2):154. [http:// cardiovascular disease: An unusual but serious complication. S Afr Med J 2015;105(2):154. [http://
dx.doi.org/10.7196/SAMJ.8638] dx.doi.org/10.7196/SAMJ.8638]

6. Stefan DC. Why is cancer not a priority in South Africa? S Afr Med J 2015;105(2):103-104. [http:// dx.doi.org/10.7196/SAMJ.9301]

7. Singh E, Underwood JM, Nattey C, Babb C, Sengayi M, Kellett P. South African National Cancer Registry: Effect of withheld data from private health systems on cancer incidence estimates. S Afr Med J 2015;105(2):107-109. [http://dx.doi.org/10.7196/SAMJ.8858]

8. Lalkhen H, Mash R. Comorbidity and multimorbidity in non-communicable diseases in South African primary healthcare. S Afr Med J 2015;105(2):134-138. [http://dx.doi.org/10.7196/SAMJ.8696]

9. Durão S, Ajumobi O, Kredo T, et al. Evidence insufficient to confirm value of screening for diabetes and hypertension in low- and-middle-income settings. S Afr Med J 2015;105(2):98-102. [http://dx.doi. org/10.7196/SAMJ.8819]

10. Hoffman K. Non-communicable diseases in South Africa: A challenge to economic development. S Afr Med J 2014;104(10):647. [http://dx.doi.org/10.7196/SAMJ.8727] 